\title{
Cervical cancer prevention in South Africa: HPV vaccination and screening both essential to achieve and maintain a reduction in incidence
}

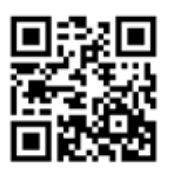

Cervical cancer remains an important cause of morbidity and mortality in South Africa (SA). A national cervical cancer prevention programme exists that offers three cervical cytology smears per lifetime, starting after the age of 30 at 10 -year intervals. Despite this programme the incidence remains unacceptably high, cases are often diagnosed late, and many patients have poor response to treatment. Primary healthcare systems in many areas are poorly developed, and uptake of cytological screening is generally poor, with some metropolitan areas and regions doing slightly better. Health systems interventions are necessary to improve the quality of screening. ${ }^{[1]}$ In addition, there is often significant loss to follow-up after the initial screening test among women identified with abnormal cytology. Determinants of the high cervical cancer rate and poor outcome of treatment are similar to those in other developing countries and include a low doctor/population ratio, a high prevalence of HIV infections, and competing healthcare needs. A lack of consumer (patient) knowledge and empowerment leads to a low degree of health-seeking behaviour.

\section{Disease prevention}

Disease prevention strategies can be broadly categorised as primary prevention and secondary prevention. Primary prevention aims to reduce the risk of an individual contracting a particular disease by eliminating the aetiological agents from the environment. In the case of cervical cancer, the most important risk factor for the development of premalignant and malignant disease is persistent infection with oncogenic types of human papillomavirus (HPV) infection.

Since the approval of the HPV vaccines in SA in 2008, they have been available in the private market. Uptake has been slow because of factors such as the initial high costs of both vaccines, poor community knowledge of cervical cancer and the causal relationship between HPV and cervical cancer, and lack of population experience with and acceptance of vaccines targeting adolescents. ${ }^{[2,3]} \mathrm{A}$ schoolbased introduction was suggested for SA. ${ }^{[4]}$ The national Department of Health introduced an HPV vaccine roll-out programme in April 2014. Introduction of the HPV vaccination programme in public schools is widely supported by scientists and healthcare workers involved in the prevention and treatment of cervical cancer, who emphasise the excellent efficacy and safety record of the vaccines. ${ }^{[5]}$

Linking health interventions may achieve cost-effective ways of preventing disease. Information from a qualitative study in SA concluded that HPV vaccination can be linked to other adolescent preventive health services. ${ }^{[6]}$ The strong link between HIV infection and immunosuppression with HPV-associated disease is well established. By controlling HIV, the incidence of HPV-related disease will also be reduced. In addition, HIV treatment facilities can be used to monitor cervical screening and treatment.

Smoking is associated with an increased risk of development of squamous and other carcinomas of the cervix, and a national antismoking campaign, like the programme introduced in SA under health minister Dlamini-Zuma, would be highly effective in reducing smoking-related diseases including cervical cancer.

Secondary prevention by screening has been shown to reduce cervical cancer significantly when comprehensive population-based call-and-recall programmes were introduced. Since the SA cervical cytology programme is not a programme of this sort and has low uptake rates, success has been limited. Opportunistic screening will continue to be an important part of our programme for the foreseeable future. Linking opportunistic screening of mothers to vaccination of their children is a potential way to increase disease awareness and screening uptake.

\section{Adolescent vaccination programmes}

The successful introduction of a school-based vaccination programme is a momentous task, especially in settings like SA where no national adolescent, adult or school-based vaccination programmes existed before roll-out. Education of healthcare practitioners and the general public will be crucial to the success of such a programme, as no framework and culture for the immunisation of older youth and adult populations are established in SA. HPV vaccine implementation programmes from other countries have shown that a school-based approach is most successful when HPV vaccines are introduced in girls $9-14$ years of age. ${ }^{[7]}$ The first of a series of three articles describing the Vaccine and Cervical Cancer Screen (VACCS) project appears in this issue of $S A M J .^{[8]}$ These pilot projects combined vaccination of adolescent girls against HPV with cervical cancer screening interventions offered to their female caregivers.

Lessons that can be learned from such vaccination pilot projects are useful to guide nationwide implementation locally and in other African countries. Barriers that had to be addressed included the challenges associated with administration of a three-dose vaccine in a busy school calendar. A two-dose schedule was investigated in phase 2 of the study in the light of new efficacy data in girls 9 - 14 years of age. ${ }^{[9]}$ The relative lack of health infrastructure for adolescent vaccination programmes was overcome by using schoolbased infrastructure and dedicated roving vaccination teams.

Parental or caregiver consent procedures could impact on vaccine uptake in school-based programmes and may be a barrier to new vaccine introduction. ${ }^{[7]}$ Clear messages to parents will reduce the likelihood of negative publicity and address concerns about safety. Vaccine uptake among girls whose caregivers attended information evenings were significantly better (almost 90\%) than that among girls whose caregivers did not attend (around 50\%), ${ }^{[8]}$ underscoring the importance of information dissemination, creating of awareness and disease-specific education. Information must be distributed using multiple strategies, which can utilise health workers or teachers. ${ }^{[7]}$ Providing written information only may not be enough in some communities. Overall, only around $50 \%$ of all invited girls were ultimately sufficiently vaccinated, mainly owing to lack of parental consent. After similar experiences, alternative strategies were employed in some countries. Community-based consent strategies that negated individual parental consent was introduced in Vietnam and Uganda, and parent opt-out strategies were used in Tanzania and Rwanda. ${ }^{[7]}$ The acceptability of mandatory vaccination in the SA context will probably be problematic.

Vaccine course completion rates were addressed. A limitation of the national HPV vaccine roll-out programme is the absence of alternative vaccination opportunities when vaccination is missed as a result of absenteeism. The VACCS trials showed that at 
least two opportunities may be needed per facility because of illness or school-related activities that could cause girls to miss the vaccination opportunity, especially if a two-dose schedule is used. HPV vaccine coverage and completion rates may also increase by the introduction of an additional public health facility-based HPV vaccination programme, which currently does not exist. The importance of grade-based as opposed to age-based eligibility criteria and completion of the vaccination series in one calendar year, as is done in the national roll-out, is highlighted by these data.

\section{Linking vaccination and screening}

An exciting and novel approach is the linking of cervical cancer screening of female caregivers to the vaccination of schoolgirls. Even though the uptake of self-screening for high-risk HPV was relatively low, a significant proportion (around 30\%) of women at increased risk for cervical cancer were identified. It is important to understand that the aim of home-based cervical cancer screening is as an adjunct to existing healthcare facility-based screening programmes to reach unscreened, high-risk women who did not access healthcare. An additional benefit of linking screening to vaccination is the ease of traceability of screened women, which decreases the loss to follow-up experienced in the current national screening programme.

Implementation of HPV vaccine programmes and extension of cervical cancer screening programmes in African countries are important steps towards reducing the high burden of cervical cancer in this region.

\section{Future considerations}

Screening with HPV testing will almost certainly replace traditional cervical cytology in the not-too-distant future. Studies to evaluate the validity of the various HPV tests in our setting are extremely important. Higher rates of HPV infection, possible differences in genotype distribution and the effects of a high background incidence will affect the performance characteristics of HPV-based screening. The possibility of patient-collected (self-sampling) specimens will cater for a large number of women who may not have access to healthcare facilities.

HPV vaccination will only make a significant impact if high enough vaccination rates can be achieved. Continuing education of the public and of healthcare workers is essential. Monitoring of the programme to evaluate vaccination rates needs to be in place from the start. A catch-up school-based vaccination campaign to include a larger cohort of girls, perhaps up to 18 years of age, will be costeffective and may produce herd protection.

\section{Conclusion}

There is currently no comprehensive cervical cancer control programme in place. We urgently need to outline strategies to:

- maximise the number of individuals receiving HPV vaccines

- monitor the vaccination programme effectively

- commit to a screening policy, with consideration of HPV testing and self-sampling.

In primary and secondary prevention of cervical cancer, opportunities for linkage with existing infrastructure and services should be investigated.

\section{H Botha}

Department of Obstetrics and Gynaecology and Unit for Gynaecological Oncology, Faculty of Medicine and Health Sciences, Stellenbosch University, Tygerberg, Cape Town, South Africa

\section{K L Richter}

Department of Medical Virology, School of Medicine, Faculty of Health Sciences, University of Pretoria, South Africa, and National Health Laboratory Service, Pretoria

Corresponding author: $M$ H Botha (mhbotha@sun.ac.za)

1. Smith N, Moodley J, Hoffman M. Challenges to cervical cancer screening in the Western Cape province. S Afr Med J 2003;93(1):32-35.

2. Adefuye PO, Broutet NJ, de Sanjose S, Denny LA. Trials and projects on cervical cancer and human papillomavirus prevention in sub-Saharan Africa. Vaccine 2013;31(Suppl 5):F53-F59. [http://dx.doi. org/10.1016/.jvaccine.2012.06.070]

3. Harries J, Moodley J, Barone MA, Mall S, Sinanovic E. Preparing for HPV vaccination in South Africa: Key challenges and opinions. Vaccine 2009;27(1):38-44. [http://dx.doi.org/10.1016/j. vaccine.2008.10.033]

4. Botha MH, Dochez C. Introducing human papillomavirus vaccines into the health system in South Africa. Vaccine 2012;30(Suppl 3):C28-C34. [http://dx.doi.org/10.1016/j.vaccine.2012.03.032]

5. Richter KL, Dreyer G, Lindeque BG, Botha MH. HPV vaccine: Can we afford to hesitate? S Afr Med J 2014;104(8):522-523. [http://dx.doi.org/10.7196/SAMJ.8449]

6. MacPhail C, Venables E, Rees H, Delany-Moretlwe S. Using HPV vaccination for promotion of an adolescent package of care: Opportunity and perspectives. BMC Public Health 2013;13:493. [http:// dx.doi.org/10.1186/1471-2458-13-493]

7. Paul P, Fabio A. Literature review of HPV vaccine delivery strategies: Considerations for school- and non-school based immunization program. Vaccine 2014;32(3):320-326. [http://dx.doi.org/10.1016/j. vaccine.2013.11.070]

8. Botha MH, van der Merwe FH, Snyman LC, Dreyer G. The Vaccine and Cervical Cancer Screen (VACCS) project: Acceptance of human papillomavirus vaccination in a school-based programme in two provinces of South Africa. S Afr Med J 2015;105(1):40-43. [http://dx.doi.org/10.7196/SAMJ.8419] 9. World Health Organization. Human papillomavirus vaccines: WHO position paper. Wkly Epidemiol $\operatorname{Rec} 2014 ; 43(89): 465-492$

S Afr Med J 2015;105(1):33-34. DOI:10.7196/SAMJ.9233 


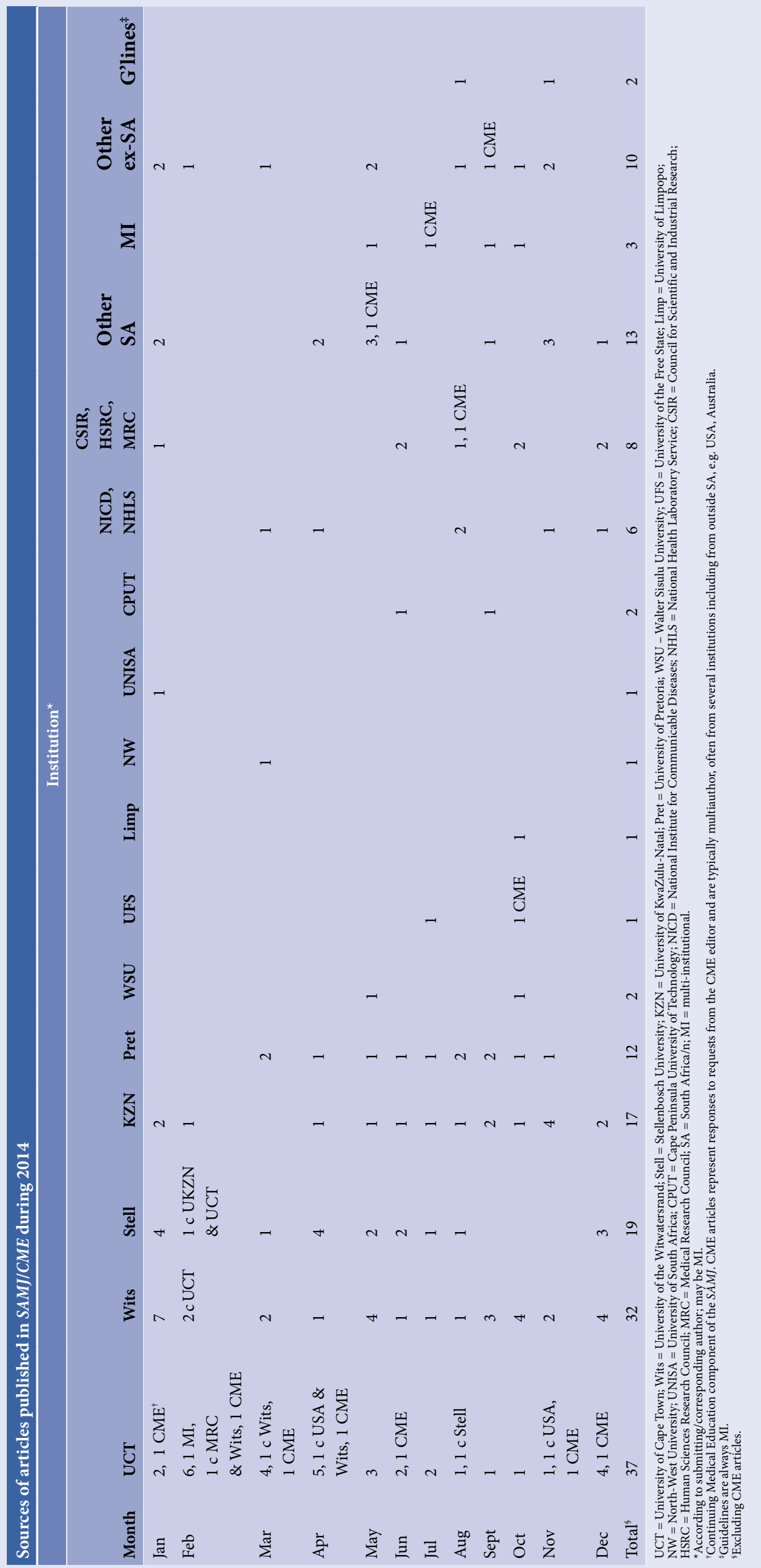

